\title{
GÉNERO Y MULTICULTURALIDAD: UN ENCUENTRO OBLIGADO. RETOS Y PERSPECTIVAS PARA LA INVESTIGACIÓN
}

\section{GENDER AND MULTICULTURALISM: A COMPELLING ENCOUNTER. CHALLENGES AND PERSPECTIVES FOR RESEARCH}

\section{Teresita Cordero*}

\begin{abstract}
RESUMEN
Se inicia el artículo presentando la contradicción que existe entre los discursos de Derechos Humanos y las condiciones de vida de las poblaciones, en el marco de un sistema capitalista, el cual no responde al ideal de sociedad que han suscrito los Estados en el nivel mundial. Esto sirve para ubicar la premisa de la diversidad, en el marco de la desigualdad $y$ las formas de discriminación social traducidas como racismo, sexismo y clasismo. Asimismo se retoman algunos avances que provienen del movimiento feminista y que ayudan a dar pistas para la investigación desde el tema de la multiculturalidad. Como conclusión general se rescatan los aportes desde una perspectiva crítica que integra el estudio de las relaciones de poder, con una concepción investigativa situada, la cual rescata las problemáticas, necesidades y demandas específicas de las mujeres en particular.
\end{abstract}

PALABRAS CLAVE: GÉNERO * CULTURA * DISCRIMINACIÓN * INVESTIGACIÓN

\section{ABSTRACT}

In the beginning of this essay, the author presents the contradiction between the discourse of human rights and the conditions of life of populations, in the environment of a capitalist system that fails to respond to the ideal of society sponsored by the world's states. This is used to place the premise of diversity in the framework of inequality and forms of social discrimination such as racism, sexism and

Instituto de Investigaciones en Educación (INIE) $y$ en el Centro de Investigación en Estudios de la Mujer, ambos de la Universidad de Costa Rica.

cordero90@hotmail.com 
classism. Some breakthroughs, that come from the feminist movement and provide insights for research from the subject of multiculturalism, are also reviewed here. As a general conclusion, the author points out the contributions from a critical perspective of power relations integrated with a situated conception of research, which in turn points to the specific problems, needs and demands of women in particular.

KEY WORDS: GENDER * CULTURE * DISCRIMINATION $*$ RESEARCH

\section{INTRODUCCIÓN}

Revisando La Declaración Universal de la UNESCO sobre la diversidad cultural y la Convención sobre la eliminación de todas las formas de discriminación contra la mujer logramos entender la existencia de principios básicos, que nos denotan y connotan un tipo de sociedad, que desde mi punto de vista está lejos de ser viable, aunque para muchos debería estar en proceso de construcción. Construcción que parece ser una realidad muy lejana a lo mejor una utopía.

Dichos principios toman en cuenta elementos cruciales que evidencian por un lado, el tema de la diversidad como una condición inherente a la especie humana y por otro, plantean la denuncia sobre la desigualdad, la discriminación y la exclusión de una sociedad caracterizada por el racismo ${ }^{1}$, el sexismo ${ }^{2}$ y el clasismo ${ }^{3}$.

1 Es la "situación de dominación y explotación de una raza por otra (o una etnia, porque hoy la palabra raza va teniendo cada vez menos valor científico) ofrece a menudo situaciones o características semejantes a las del sexismo..." (Sau, 1990: 255).

El sistema patriarcal está íntimamente ligado al concepto denominado sexismo, pues se entiende por "Patriarcado: Construcción social en la cual los hombres y sus acciones son más valoradas socialmente que las de las mujeres, a quienes se oprime y explota. El término considera las estructuras de poder $y$ la violencia para mantenerlo. La discriminación por sexo, propia de este sistema, se denomina sexismo" (González, 2008. Artículo en proceso, p. 2).

Posición discriminatoria que mantiene las divisiones de clase social, ubicando a unas y otras como superiores y por lo tanto legitimando las relaciones de poder.
Es interesante que en la actualidad nos encontremos con una comunidad de seres humanos interconectada como nunca antes. Pero a su vez sujeta a una serie de condiciones $y$ contradicciones cuyas consecuencias se viven cotidianamente.

Hallak (1999) plantea como la globalización en las sociedades humanas son el resultado de la integración de los sectores económi$\cos y$ financieros en el nivel mundial, lo que hace que existan poderes paralelos a los de los Estados Nacionales, cuya presión ha ido debilitando a los gobiernos $y$ a los proyectos de sociedad que se han sustentado. Dichos poderes, no responden necesariamente a los intereses de lo que se promulga en las declaraciones sobre los Derechos Humanos.

Asimismo, Espeja, Chaves y Robles nos indican que la "lógica capitalista se agudiza con el avance tecnológico incidiendo en la vida socio cultural, ya que se caracteriza por la existencia de un proceso de unificación mundial, pero que no reviste la atmósfera de comunidad y menos de aldea", son las de una "Economía tipo Madonna, es decir, un proceso de unificación del consumo de los bienes de información y de comunicación que se levanta sobre una misma lógica, la del mercado capitalista $y$ hace referencia a los mismos recursos - las infraestructuras y las redes de publicidad masiva de dimensión planetaria" (1999: 64). Cuyo énfasis está marcado por una ideología dominante que excluye concepciones y visiones de mundo no coincidentes.

Aunado a este marco general encontramos que el racismo, el sexismo y el clasismo tienen en común que son expresiones de las formas de dominación $y$ de explotación sobre grupos humanos. La defensa y el derecho a la diversidad y la no discriminación apoyan 
el reconocimiento a la multiculturalidad y de temáticas y condiciones sobre la vida de las mujeres.

Surgen entonces las voces de las minorías, entendidas estas no por la cantidad, sino por ser grupos excluidos de los beneficios, las oportunidades que la especie humana como sociedad ha logrado.

\section{GÉNERO Y MULTICULTURALIDAD}

La perspectiva de género, en el marco del feminismo y como categoría analítica y política comprende que es posible cambiar las relaciones de desigualdad, dominación y discriminación, porque somos seres construidos socialmente (González, 2008). Y con categoría no se está pensando acá en hombre y mujer, esto va considerado como una fuente de análisis integral, donde las condiciones de vida, la pertenencia a una clase y etnia, las oportunidades y los obstáculos de una práctica cultural particular posibilitan o limitan el pleno desarrollo como seres humanos. Así la categoría género se reviste de una perspectiva ética política que en la investigación social describe, analiza, interpreta y comprende sin perder de vista la crítica que tienen algunas propuestas feministas.

Las posturas feministas han permitido ir deconstruyendo $y$ denunciando las formas de relación desigual en esta sociedad, centralizadas en y desde las mujeres. Esto ha implicado la discusión de temas de gran impacto social como por ejemplo: la división sexual del trabajo, la sexualidad, la violencia como eje de dominación y las relaciones de poder, tan solo para citar algunos.

En este proceso de evolución del pensamiento feminista, $y$ no necesariamente de las feministas, el eje de la diversidad se vuelve una perspectiva que nos lleva a la multiculturalidad. Conceptos que parecen remitirnos a dos ideas que tenemos que revisar. Críticamente, por una parte, el relativismo cultural y por otra, la idea de la "cultura" como un grupo social segregado y que no se integra a las costumbres de otros grupos sociales, como si esto fuera posible.
1. Con respecto al relativismo cultural, es claro que en todo grupo humano existe una historicidad propia y particular, que nos permite significar $y$ hacer actividades de manera distinta, la creatividad humana es extraordinaria. Esta idea por supuesto choca contra la idea de universalidad y con la búsqueda, en el caso de ciencia, de las generalidades. La tensión suele expresarse en la investigación social cuando se adoptan posturas epistemológicas y metodológicas de las que se han llamado investigación cuantitativa o cualitativa. Detrás de la idea del relativismo está el dejar pasar y dejar hacer sin aparentemente cuestionar los mecanismos de dominación y explotación que hay al interior de los grupos sociales. Ya que podemos decir que hay prácticas culturales distintas pero también, debemos revisar las relaciones de poder que hay al interior de los grupos humanos: quiénes se benefician, qué tipo de privilegios se dan y qué estructuración social se sostiene. Esta postura nos lleva a realizar un encuentro obligado del género con la multiculturalidad.

2. Por su lado la idea de la segregación, en un mundo cada vez más interrelacionado, se sustenta sobre el mito de la no comunicación y la no convivencia intercultural. Por supuesto que existen estudios diversos sobre los mecanismos de resistencia de grupos migrantes, indígenas $y$ otros esfuerzos por mantener las costumbres $y$ las tradiciones de sus grupos de pertenencia, pero esto no significa que no se integren prácticas culturales diferentes a las propias. El rescate de las raíces y la historia son fundamentales en la construcción de la identidad personal y grupal. No obstante, considerar al getho social es una forma de dominación. Recordemos el colonialismo, una experiencia que nos marca lo que hoy somos en nuestra historia, en nuestra psique $y$ en nuestra manera de considerar las formas de relación social. Esta etapa de vida que proviene de más de 500 años, no solo devasta a grupos enteros, sino que 
desde una ideología racista estructuró prácticas de dominación y explotación que hemos heredado y que se constituyen en actuaciones cotidianas, que se evidencian en la señora campesina que reniega de su color de piel $y$ que añora $y$ valora la blanquitud en su supuesta superioridad.

\section{APORTES DEL FEMINISMO QUE SE INTEGRAN CON LA PERSPECTIVA MULTICULTURAL}

Sampson nos indica que el análisis feminista ha permitido comprender “... que los grupos dominantes se definen en términos de sus intereses, sus deseos y sus temores" (1996: 38 ), en este sentido los aspectos economicistas $y$ la lógica de un mercado que regula la vida humana prevalece sobre la construcción de una sociedad más justa y respetuosa de los Derechos Humanos sobre todo aquellos económicos, culturales y ambientales. La crisis alimentaria es tan solo un ejemplo del proceso de deshumanización de la vida humana y de la irracionalidad de un sistema que tiende a la destrucción.

Asimismo especifica "que el tener voz no implica meramente la posibilidad de hablar, sino también el registro y la forma en que discurre este hablar" (Sampson, 1996: 38) no basta con poder hablar sino que es importante tener el espacio de contar con oportunidades $y$ garantizar los beneficios que como sociedad hemos construido. En días pasados nos comentaba la profesora Miriam Maldonado (Cordero, 2008) que en la Universidad de San Carlos de Guatemala, recientemente, ha introducido una prueba de admisión que incluye solo el lenguaje español, en un país multiétnico y multilingüe. Esto ya implica un obstáculo y una forma de discriminación para la población indígena guatemalteca, en especial para las mujeres en lo que respecta el acceso a la educación superior pública.

Continúa el autor planteando que los estudios feministas han logrado demostrar que "las dinámicas de dominación tienen una larga historia” (Sampson, 1996: 38). La construcción social del género es el producto de una sociedad patriarcal que ha venido sustentando prácticas, ideas $y$ concepciones legitimadas por las pro- pias instituciones sociales, entre ellas la religión, los medios de comunicación social y las leyes, para tan solo mencionar algunas.

De manera interesante y rescata la premisa que "... argumenta que los objetos de la cultura, incluyendo el objeto que aparentemente es el más natural de todos, el sexo de una persona, se construye discusivamente" (Sampson, 1996: 38). El fenómeno de la naturalización de la vida cotidiana y la legitimización de los discursos dominantes son uno de los mecanismos más fuertes para mantener la dominación. El racismo y la segregación por el color de la piel son algunos ejemplos que se pueden integrar con el del sexo biológico y ni que decir de la violencia sexual que ha sido una práctica de dominación. En un reciente estudio de Castro que realiza con mujeres negras costarricenses entre sus conclusiones expone como el género y la raza se traduce en el cuerpo:

... El cuerpo además de definir es un medio por el que se canaliza el control social a través de diferentes formas de agredirlo y enmarcarlo. Por medio del cuerpo se busca un sometimiento, pero por medio del cuerpo se inicia, recordando los discusos de las mujeres negras, 'la batalla'. En el caso de las mujeres negras interfieren las variables del género y la raza de manera dialéctica agrupándolas (2006: 100).

Agregando a lo planteado se considera entonces que los estudios multiculturales también deberían coincidir con los postulados del feminismo en cuanto que luchan contra la dominación y la discriminación social.

Esta opresión como nos menciona Gómez citando a Teresa de Laurenti:

En los análisis feministas, el concepto de sujeto femenino descansa en la noción de una opresión homogénea de las mujeres, aún cuando sabemos que no es la misma situación de vida de las mujeres burguesas que la de las mujeres proletarias, ni la situación de las mujeres negras o indígenas puede ser asimilada a una noción 
general como La Mujer. Tampoco podemos obviar los factores de edad o no considerar los ciclos de vida de las mujeres si pretendemos realmente comprender su posición en la cultura (2002: 71).

\section{RETOS Y DESAFÍOS PARA LA INVESTIGACIÓN SOCIAL}

Desde la investigación uno de los puntos más críticos para el o la investigadora, son los sesgos patriarcales sobre los que hemos sido socializados sobre lo que es ser hombre $y$ lo que es ser mujer, relacionados con la división sexual del trabajo, del tipo de oportunidades y responsabilidades que se asignan, las formas de ejercer el poder, la invisibilización de las necesidades, entre otros aspectos más.

Así, nuestras propias percepciones, ideas y significados que construimos como sujetos humanos influyen en la investigación que hagamos, en los intereses que tengamos como clase privilegiada, que tiene el tiempo $y$ las condiciones para acceder al conocimiento. De manera paradójica, la oportunidad de estudiar y revisar diferentes perspectivas teóricas, conceptualizaciones $y$ visiones de mundo nos da la oportunidad de transformar nuestra propia visión que se puede traducir en el trabajo que realizamos. Esta oportunidad nos demanda una respuesta crítica a nuestros trabajos y a los eventos que investigamos como una práctica permanente.

Hernández plantea la crítica al feminismo hegemónico que desde su punto de vista, no había logrado integrar la visión de las mujeres indígenas por responder a otra agendas $y$ necesidades de grupos más vinculados con la vida de la ciudad:

Por un lado, las mujeres indígenas organizadas han unido sus voces al movimiento indígena nacional para denunciar la opresión económica y el racismo que marca la inserción de los pueblos indios en el proyecto nacional. Paralelamente estas mujeres están desarrollando un discurso y una práctica política propia a partir de una perspectiva de género situada culturalmente, que viene a cuestionar el sexismo y el esencialismo de las organizaciones indígenas, como el etnocentrismo del feminismo hegemónico (2001: 207).

Es necesario por lo tanto, considerar en la investigación el cuestionamiento de las visiones esencialistas sobre las mujeres $y$ ciertos grupos étnicos o sociales particulares, así como las ideas de generalizaciones que provienen del feminismo que no han considerado la etnia y la clase social.

Esto nos lleva a plantear la necesidad de realizar una práctica investigativa que pudiera apoyar una agenda amplia que no olvide el racismo, el sexismo, el clasismo, como parte de su reflexión y análisis.

En un recuento somero, el informe de la reunión de expertas sobre racismo y género de las Naciones Unidas de la CEPAL en el año 2001, nos detalla una amplia agenda para la academia $y$ que procedo a resumir:

1. Pacarí sugería incluir y discutir las categorías de discriminación racial, género y clase (citado en CEPAL, 2001).

2. Galván recomendaba la necesidad de repensar las formas teóricas de análisis sobre los ejes de género y multiculturalidad (citado en CEPAL, 2001).

3. Santana por su parte consideraba que desde la historia había que seguir estudiando la colonización (citado en CEPAL, 2001).

4. Peredo la servidumbre desde una visión de la otredad pero a partir del déficit, los otros que les falta, los incivilizados y demás. Por ello aportaba que es requisito estudiar $y$ analizar las formas de discriminación y los procesos de subordinación (citado en CEPAL, 2001).

5. Alvarado la necesidad del reconocimiento de los conocimientos tradicionales como la medicina, la cultura y el arte (citado en CEPAL, 2001).

6. Rivera por su parte, la vinculación de los grupos indígenas y negros en relación con las condiciones de pobreza y miseria en que 
se encuentran. Esto la lleva a considerar los marcos legales multiculturales (citado en CEPAL, 2001).

7. Hernández planteaba el fortalecimiento de las relaciones interculturales para romper la subvaloración de las lenguas indígenas (citado en CEPAL, 2001).

A manera de ejemplo se retoman dos trabajos elaborados por el Instituto Nacional de las Mujeres de Costa Rica (INAMU) que evidencia la situación de las féminas de los grupos indígenas.

El INAMU (2007b) en la investigación que realiza, retoma las historias de vida de mujeres indígenas y plantea como conclusiones generales:

$\diamond$ Reconocer la participación de las organizaciones femeninas en el marco de la defensa de los derechos humanos de los pueblos autóctonos.

$\diamond$ Las mujeres indígenas enfrentan problemas de violencia de género, por el solo hecho de ser mujeres, unido a la discriminación de una sociedad excluyente.

La violencia, violencias $y$ violentaciones son parte de las cotidianidades de las mujeres, aquí urge canalizar las denuncias presentadas por las mujeres indígenas en sus testimonios (INAMU, 2007b: 84).

$\diamond$ La necesidad de reconocer las particularidades de las mujeres de cada pueblo y no pretender homogenizarlas. Reconocer las diversidades.

$\diamond$ Realizar investigaciones para llenar el vacío de las investigaciones en Costa Rica.

$\diamond$ Reconocer las condiciones de vulnerabilidad de las poblaciones en especial con respecto a la vida cotidiana de las mujeres.

Sintetiza el estudio:

Los mandatos patriarcales naturalizan la desigualdad, la exclusión, la marginalización y la invisibilización. Urge crear nuevas socializaciones desde los derechos pero con prácticas culturales transformadas que respeten a las mujeres (INAMU, 2007b: 84).
Así en otro documento el INAMU (2007a) recoge las demandas de las mujeres indígenas relacionadas con el trabajo, la prevención de la violencia, la capacitación, apoyo en el cuidado infantil, acceso al financiamiento y atención de las dolencias, entre muchas más. Temas que no están lejanas de las demandas de las mujeres en general.

Como puntos en común podemos decir que es necesario visibilizar las demandas de las mujeres, que son la mitad de la población y evidenciar los procesos de dominación, subordinación y discriminación de género de manera situada, en sus propios grupos sociales de referencia. Esto significa visualizar la especificidad de los mecanismos de dominación y violencia contra las mujeres.

Además, no caer en un idealismo de respetar costumbres $y$ tradiciones en un acto de dejar hacer, dejar pasar; $y$ más bien poder crear condiciones para la reflexión al interior de los grupos sociales sobre sus condiciones de vida y sobre los mecanismos de exclusión social que viven. Para cerrar retomo una frase que escribe Hernández desde un grupo de mujeres indígenas:

También tenemos que pensar qué se tiene que hacer nuevo en nuestras costumbres; la ley sólo debería proteger y promover los usos y costumbres que las mujeres, comunidades y organizaciones analicen si son buenas. Las costumbres que tengamos no deben hacer daño a nadie (2001: 218-219).

\section{BIBLIOGRAFÍA}

Castro Carmiol, Evelyn. Representaciones contemporáneas de la exclavitud en mujeres afrocostarricenses desde sus propias voces. Chile: Universidad de Chile. Facultad de Ciencias Sociales. Escuela de Postgrado Magíster en Estudios de Género y Cultura. Mención Ciencias Sociales, 2006.

Cobo, Rosa. "Multiculturalismo, democracia paritaria y participación política". Política y Sociedad 32. Madrid, 1999. En: $<w w w . i i d h . e d . c r-B i b l i o t e c a w e b>$ [fecha de acceso: 11/03/2008]. 
Cordero, Teresita. Comunicación personal con Miriam Maldonado. En el Encuentro Interamericano sobre Género y Gobernabilidad. Córdoba, Argentina. 24 al 26 de abril, 2008.

Espeja, Jesús; Chaves, Jorge A.; Robles, Armando. Transformación cultural, economía y evangelio. España: Editorial San Esteban, 1999.

Gómez Campos, Rubí de María. Género y multiculturalidad. DEVENIRES III (5). 2002: 67-80.

González, Mirta. Feminismo(s) Avances hacia la equidad. Documento borrador para libro. 2008.

Hallak, Jacques. Globalización, derechos humanos y educación. Paris: UNESCO. Instituto Internacional de Planeación de la Educación, 1999.

Hernández Castillo, R. Aída. "Entre el etnocentrismo feminista $y$ el esencialismo étnico. Las mujeres indígenas y sus demandas de género". Debate feminista, racismo, mestizaje 24. Año 12 . México, octubre 2001.

INAMU. Las mujeres indigenas en Costa Rica: un acercamiento a su realidad $y$ propuestas de acción. Mesa Nacional Indígena. Comisión de Mujeres Indígenas Warë Kané. Colección Documentos
41. San José: Instituto Nacional de las Mujeres, 2007a.

INAMU. Mujeres Indigenas Costarricenses: los procesos de creación de sus organizaciones. Colección Documentos 46. San José: Instituto Nacional de las Mujeres, 2007b.

Naciones Unidas, CEPAL. Informe de la reunión de expertas sobre racismo y género. Santiago de Chile: 4 y 5 de junio. Documento 27 noviembre, 2001.

Peredo Beltrán, Elizabeth. Naciones Unidas, CEPAL.Una aproximación a la problemática de género etnicidad en América Latina. Santiago de Chile: 4 y 5 de junio. Documento 4 junio, 2001.

Sampson, Edward E. "Celebrar al otro. Una interpretación dialógica de la naturaleza humana". En: Cordero, Teresita; Dobles, Ignacio; Pérez, Rolando. Dominación Social y Subjetividad. Contribuciones de la Psicología Social. San José, Costa Rica: Editorial de Universidad de Costa Rica, 1996.

Sau, Victoria. Diccionario ideológico feminista. Bacerlona: ICARIA Editorial, SA., 1990.

UNESCO. Convención sobre la eliminación de todas las formas de discriminación contra la mujer. Declaración Universal de la UNESCO sobre Diversidad Cultural. 